\title{
Antonio Ibarra, Mercado e institución: corporaciones comerciales, redes de negocios y crisis colonial: Guadalajara en el siglo XVIII, México, Universidad Nacional Autónoma de México/Bonilla Artigas Editores, 2017, 376 pp.
}

\author{
Sebastián Porfirio Herrera Guevara*
}

15 de marzo de 2018

A obra titulada Mercado e institución: corporaciones comerciales, redes de
negocios y crisis colonial. Guadalajara en el siglo XVIII, consiste en una
compilación de doce textos que en su conjunto aborda una variedad de temáticas
como lo son las discusiones historiográficas que permean en la historia económica
reciente; el análisis del mercado interno novohispano a partir del caso de Guada-
lajara, así como sus alcances y límites; también, la participación del Consulado
como un elemento de antagonismo corporativo con otros grupos de comerciantes,
entre otros temas.

Desde un punto de vista general, estos trabajos publicados en revistas y libros durante las décadas de 1990 y 2000, tienen como eje rector la Guadalajara tardocolonial, particularmente aquella de finales del siglo XVIII y principios del XIX. Lo interesante es que no se trata de textos que busquen la endogamia, como aportaciones locales a una historiografía que se mira en el espejo, sino que hay un esfuerzo por trascender esa barrera. Así, a partir del estudio de esta región, Antonio Ibarra propone una serie de discusiones de diferente calado que profundiza en el conocimiento, la metodología y las aportaciones de la historia económica. En ese sentido, Guadalajara es un estudio de caso que le permite al autor dialogar y discutir, siempre en términos amistosos, con Ruggiero Romano, o bien tomar el caso del Consulado para aclimatar los postulados de Douglas North y los neoinstitucionalistas. Dicho en palabras del autor lo que se pretende evidenciar es: "cómo

\footnotetext{
*Universidad de Guadalajara. email: sebastian@colmich.edu.mx
} 
en cada espacio los actores [locales] resolvieron su inscripción en los procesos globales" (p. 31). Es decir, la relación dialéctica que existe entre ambos. Visto de este modo, el presente volumen, en última instancia, es el reflejo de una trayectoria académica sólida que se manifiesta en el abordaje de temáticas complejas e intereses diversos.

Puntualmente, existen dos ejes que transversalmente se abordan a lo largo de los capítulos de la obra, el primero tiene que ver con la configuración y alcance del mercado de Guadalajara, en tanto el segundo se centra en las instituciones económicas como elementos útiles para explicaciones de corte histórico, pero no elaboradas de una forma rígida, sino haciendo énfasis en la participación los actores en estos procesos.

El primer texto (capítulo I) es un balance historiográfico a partir del cual se sientan las bases para las discusiones que se abordarán a lo largo del volumen. Se indica la preeminencia de trabajos centrados en el periodo colonial, en gran parte debido a un revisionismo que ha cuestionado las ortodoxias teóricas, al tiempo que ha abierto el camino para modelos de explicación más rigurosos. Aún así, se ponen de manifiesto las aportaciones señeras de una historiografía, por ejemplo de los precios, que sentó las bases para estudios mucho más maduros centrados en el aspecto financiero, la historia fiscal, la industrial o la empresarial. En ese sentido, el enfoque institucional resulta útil para la explicación histórica y tuvo una buena recepción en diversos trabajos monográficos que se publicaron a partir de la década de 1990, a partir de esa renovación teórica y metodológica, de la cual el autor indudablemente se alimenta, comienza este recorrido por comprender desde diferentes matices la región económica de Guadalajara.

Siguiendo el recorrido del libro, nos encontramos con uno de los primeros debates referente a la organización de un mercado regional, y que constituye los siguientes dos capítulos (II y III). Como es conocido existe una serie de trabajos que apoya la idea de la insularidad económica de la Guadalajara tardocolonial (el principal exponente es Eric Van Young). Tales trabajos delinean una sociedad que produce riqueza pero que deja a ciertos mercados internos funcionando con una economía natural o muy poco monetarizada, en la cual las distancias juegan un papel central en términos de aislamiento. Contrario a ello, Ibarra propone que la economía regional era abierta y dinámica. En la transición de una economía que se miraba en el espejo, durante el siglo XVIII se imbricaron procesos en una serie vinculada al crecimiento económico, como el aumento de la producción agrícola para satisfacer la demanda de la ciudad y su área de influencia, la cual experimentaba un aumento poblacional evidente.

Pero la importancia de Guadalajara y su región no se sustenta únicamente en estas variables, sino en una economía que logró diversificarse a través de una integración con otros mercados, especialmente a través de productos especializados. En última instancia se trata de un círculo virtuoso. Este enfoque extraregional 
es sumamente interesante pues se vincula, a través del estudio de la demanda, a una serie de actores e instituciones en un contexto que tenía alcances que iban más allá de lo tradicionalmente previsto. Estas afirmaciones se sustentan en fuentes que son reinterpretadas con mucho rigor, por ejemplo, en el uso que se le da al documento del intendente Abascal y Souza, una fuente de mucha riqueza en cuanto a datos, a partir de la cual el autor puede sustentar una lectura distinta de la estructura productiva regional.

Esta economía articulada en sectores mantuvo como base el abasto agrícola para el mercado interno, pero se diversificó hacia el sector externo. En ese sentido, ¿realmente se trataba de un mercado carente de circulante para el comercio interior? Aquí se integra el debate con Ruggiero Romano, el cual destaca por la apertura a mantener un diálogo que resulte fructífero. En un ámbito donde las ortodoxias suelen ser lo común, el disenso y el señalamiento sin duda son acciones saludables para cualquier historiografía y aún más cuando existe una retroalimentación que se mantiene en el mismo tenor. Del diálogo se rescata la reflexión sobre el uso riguroso de la fuente cuantitativa, así como de los alcances interpretativos que esta puede tener.

El otro eje del libro, el cual abarca transversalmente el resto de los capítulos, consiste en investigar la participación de los diversos actores sociales en relación con las instituciones económicas. En este sentido, la pregunta de fondo es la sustentabilidad o el fracaso de las instituciones, así como la ponderación del accionar individual en su funcionamiento y desarrollo cotidianos, es decir, centrarse en cómo los actores llevaban a cabo sus negocios. Si bien se puede pensar que los actores importan más que las instituciones, estas también son fundamentales para el accionar de los primeros, pues les proveen de marcos de normativos y de acción. Así, el autor menciona que: "actor y corporación constituyen una tensión crítica que se resolvía en la negociación política o en los acuerdos de mercado" (p. 33).

El primer texto que versa sobre este segundo eje (capítulo v) aborda la feria de San Juan de los Lagos como un evento que ayudó a articular a la clase comerciante tapatía con un mercado extrarregional de amplio alcance, pues los vínculos comerciales que llegaba a tener la feria eran realmente anchos en términos geográficos. Fue la plataforma política perfecta para que se manifestaran los actores locales quienes ganaron influencia en aspecto territoriales, fiscales y comerciales. La afirmación anterior se logra nuevamente con un estudio riguroso de fuentes cuantitativas que permiten tener una panorámica del comercio que se llevaba a cabo en la feria por varios años.

En los siguientes capítulos (V, VI y VII) el autor se concentra en la participación del Consulado en la disputa por el control de las importaciones. Aquí es importante resaltar el enfoque centrado en los actores, que los consulados tienen que ver con la acción de grupos concretos, los cuales estaban inmersos en un 
momento de apertura comercial. En un contexto de reformas orientadas al crecimiento económico, la elite orientó sus esfuerzos a obtener el mayor grado de influencia y ganancia. En ese sentido, el accionar del Consulado derivó en la noción de cohesión mercantil que ayudó a la "dilatación espacial del mercado interno novohispano" (p. 170), pero también a tener un accionar coherente y sistemático en términos de grupo. El estudio de caso se centra en Guadalajara y Veracruz, dos experiencias tardías en comparación con la de México. Particularmente a través de la sistematización de los cobros de avería, se puede ponderar la importancia del mercado interno y en particular la centralidad de Guadalajara como el vértice que vinculaba la costa con tierra adentro, la reconstrucción de estas rutas permite vislumbrar que los alcances hacia el septentrión novohispano eran considerables. En suma, el gremio de comerciantes creó una estrategia comercial y corporativa, a partir de la obtención de instrumentos institucionales, que le permitió como grupo tener soberanía sobre un territorio y un mercado.

Por supuesto, el autor lleva el análisis hacia los actores mostrando un consulado controlado por una elite comerciante que actuó en términos colectivos, con un amplio sentido gregario que le permitió consolidar una capacidad de gestión muy relevante en detrimento de ciertos mayoristas de la capital. Ibarra destaca un doble proceso aparentemente opuesto, por un lado la fragmentación de la soberanía de grupos comerciantes al entrar en el juego Veracruz y Guadalajara, pero al mismo tiempo un comportamiento corporativo que llevó a consolidar intereses comunes. Fragmentación y cohesión, así era la política dentro de la crisis imperial, una mezcla de elementos de antiguo régimen, por su carácter corporativo, con otros que se vinculan ya a una concepción moderna, especialmente debido a la gestión institucional. Esta dualidad conflictiva estribaba en que eran instituciones que pretendieron establecer la modernidad borbónica, especialmente en lo relativo al libre comercio. Por supuesto, gran parte de esta influencia se logró a través del intercambio de información privilegiada dada por nexos familiares y enlaces matrimoniales. El estudio de esta elite permitiría abrir luces sobre los cambios institucionales y en última instancia políticos que se manifestaron en la época.

Ahora bien, cabe preguntarse que tanto la ampliación de estos consulados derivó realmente en una oposición franca entre elites comerciantes o si fue en realidad una ampliación y diversificación de su influencia hegemónica. El siguiente apartado del libro (capítulo VIII) da cuenta de esta pregunta, primero se delinea nuevamente (a través de fuentes fiscales) un mercado dinámico y vinculado hacia afuera, en el cual, en palabras de Ibarra: si bien "el autonomismo era una buena herramienta de negociación, pero no una inteligente actitud ante el mercado y la economía novohispana de la época" (p. 222). En ese sentido, el sentido corporativo estaba presente en muchos de los conflictos existentes, ya sea, por ejemplo, por la jurisdicción territorial o cobro de derechos de circulación. El caso es que pese a las tensiones, los lazos comerciales eran en ocasiones muy fuertes y se 
venían dando desde años atrás beneficiando a sus partes integrantes, es posible que esta articulación de intereses colectivos tuviera un peso mayor en el desarrollo cotidiano de la actividad comercial en la época. Lo anterior viene a matizar el carácter estático que suele dársele a la configuración de redes empresariales, ponderando el hecho que los individuos que las conforman suelen actuar por razones más complejas que las evidentes, en ocasiones llevados por aspectos emocionales o de pertenencia más que racionales.

Sin duda, los primero beneficiarios eran los dirigentes de los nuevos consulados; pero a través de este comportamiento corporativo se creó una dinámica de ganancia que benefició e incorporó a otros actores pertenecientes a la clase comerciante, pero que no tenían la misma preeminencia, aún así la creación de redes de contactos permitió ampliar el número de asociaciones, lo que devino en un detrimento de los monopolios previamente establecidos. Lo anterior no quiere decir la desaparición de un grupo de elite dominante por otro, sino la rearticulación de los ciclos de comercio y, por ende, la reconfiguración de las áreas de influencia y poder dentro de las cúpulas dominantes.

En ese contexto, el comercio persistió, se diversificó y se consolidó. Un ejemplo de ello es el análisis del consumo de efecto orientales en el mercado interno regional, el cual era amplio y variado según la calidad y uso social de quienes los adquirían, y que implicaba: porcelanas, biombos, seda, entre otros productos. Estos enlaces no se explican sino a través de complejas redes comerciales que permitían la circulación de estos productos en el mercado interior novohispano. En ese sentido, las gráficas de reconstrucción de redes son una forma de plasmar el esqueleto de un entramado que era muy complejo y que se explica más allá de las desiciones de los dirigentes consulares. Un caso similar sucede con la articulación que existió entre los consulados de Guadalajara y Buenos Aires. El de desarrollo de estos apartados constituye los capítulos IX y X.

Por último se plantea problemáticas sobre la modernidad y la rebelión, capítulos XI y XII, en un contexto en el cual las nociones de legalidad y legitimidad se pone en entredicho, los tradicionales marcos mentales corporativos persistieron en detrimento de un reforzamiento de una cultura de credibilidad por parte del Estado, estas crisis profundas tendrían enormes repercusiones en el largo y difícil proceso de consolidación estatal experimentado en el país durante el siglo XIX.

En suma, considero que la conjunción de textos que se presenta en el presente volumen constituye un aporte valioso para la historia económica pues abunda en conocer la conformación de mercados integrados, también es una obra interesante para los interesados en observar la aclimatación de los postulados institucionalistas en estudios de caso regionales. No obstante, creo que el rigor en el uso de las fuentes y el fino entramado que se hace a partir de ellas permite también comprender procesos que tocan a historiadores sociales y político. Esa apertura es la que más celebro de este volumen, pues el autor no busca encerrarse en un saber 
sofisticado, y en muchas ocasiones difícil de comprender para la mayoría de nosotros, sino que tiende puentes para el diálogo, sólo falta quién le tome la palabra. La invitación a ese diálogo está puesta a través de este libro. 